\title{
Microscopic Dielectric Response Functions in Semiconductor Quantum Dots
}

\author{
Xavier Cartoixà and Lin-Wang Wang \\ Computational Research Division, Lawrence Berkeley National Laboratory, Berkeley, California 94720, USA
}

(Received 10 January 2005; published 15 June 2005)

\begin{abstract}
We calculate and model the microscopic dielectric response function for quantum dots using first principle methods. We find that the response is bulklike inside the quantum dots, and the reduction of the macroscopic dielectric constants is a surface effect. We present a model for the microscopic dielectric function which reproduces well the directly calculated results and can be used to solve the Poisson equation in a nanosystem.
\end{abstract}

DOI: $10.1103 /$ PhysRevLett.94.236804

PACS numbers: 73.22.-f, 73.21.-b

The dielectric response function is of paramount importance in nanoscience. It has been found both theoretically [1-3] and experimentally [4] that the averaged dielectric constants of small (1-5 nm) quantum dots (QD) are significantly reduced from their bulk values. Originally, this reduction was attributed to the band gap increase inside the quantum dot. According to this theory, the dielectric response at the interior of the quantum dot should be reduced compared to its bulk value. However, recently, Delerue et al. [5] have resorted to a theorem by von Laue [6,7] to argue that the influence of the quantum dot boundary should only be felt close to the surface, hence screening away from it should be bulklike, and therefore the reduction in the averaged dielectric constant is only a surface effect. They have performed empirical tight-binding calculations for test cases that validated their arguments for the averaged macroscopic response functions (e.g., under a uniform external electric field). This controversy raises a serious question: What is the correct microscopic dielectric function to be used in a nanosystem? Note that, in a phenomenological classical description, the effective macroscopic dielectric screening for the electron-hole interaction inside a QD is also bulklike due to a cancellation of a self-interaction energy and a surface induced interaction term $[8,9]$.

Here, using $a b$ initio calculations, we will study and model the microscopic dielectric function in a nanosystem. We will focus on the microscopic charge response function $\chi\left(\mathbf{r}_{1}, \mathbf{r}_{2}\right) \equiv \delta \rho\left(\mathbf{r}_{1}\right) / \delta V_{\text {tot }}\left(\mathbf{r}_{2}\right)$, where $\delta \rho\left(\mathbf{r}_{1}\right)$ is the change in the charge density due to a change in the total (external + induced) potential $\delta V_{\text {tot }}\left(\mathbf{r}_{2}\right)$. The dielectric function $\epsilon\left(\mathbf{r}_{1}, \mathbf{r}_{2}\right)$ equals $1+4 \pi \int \chi\left(\mathbf{r}, \mathbf{r}_{2}\right) /\left|\mathbf{r}_{1}-\mathbf{r}\right| d^{3} \mathbf{r}$.

We first calculate the charge density response in a 933atom GaAs QD to a $\delta$-like perturbation localized at the center of the dot. The calculation is done in the local density approximation (LDA) of density functional theory using norm-conserving pseudopotentials and a plane wave basis set with a 25 Ry kinetic energy cutoff. The charge density response is calculated by the difference of the total non-self-consistent charge densities with and without the perturbation potential. The surface of the QD is passivated by hydrogenlike atoms with partial charges [10], which resemble real passivations filling all the surface dangling bonds. The same response function is calculated for the bulk using a 1000 -atom periodic cubic supercell.

Figure 1(a) shows a (001) cross section in the charge density response $\Delta \rho_{d}(\mathbf{r})$ for the 933 -atom (including surface passivation atoms) QD. If the corresponding bulk charge density response $\Delta \rho_{b}(\mathbf{r})$ was plotted on top of $\Delta \rho_{d}(\mathbf{r})$ in the same figure, one would not be able to see any difference. Thus, we have shown in Fig. 1(b) the difference $\Delta \rho_{d}-\Delta \rho_{b}$. Noticing the scale difference, $\Delta \rho_{d}-$ $\Delta \rho_{b}$ is only $0.3 \%$ of $\Delta \rho_{b}$ at the center. The same is true between $\int\left|\Delta \rho_{d}-\Delta \rho_{b}\right| d^{3} r$ and $\int\left|\Delta \rho_{b}\right| d^{3} r$. This small difference could be due to technical details, such as different $\mathbf{k}$-point samplings.

The above data are presented in a different form more suitable for analysis in Fig. 2(a). This form will be used throughout the rest of the Letter. The curves show the spherical averaged $\Delta \rho(r)=r^{2} \int \Delta \rho(\mathbf{r}) d \Omega$, i.e., the charge density within a shell of $r$ and $r+d r$. Again, we observe
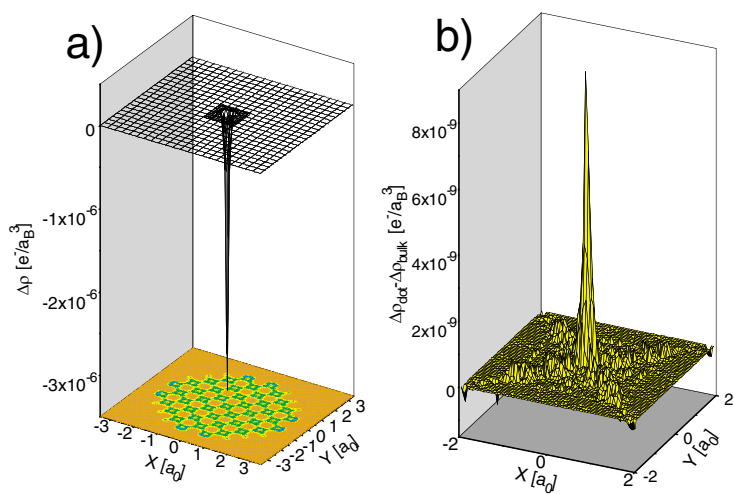

FIG. 1 (color online). (a) Cross section of the change in the charge density of a 933-atom GaAs quantum dot due to a $\delta$-like perturbing potential. The full grid is only used in the small central region with significant $\Delta \rho_{d}(\mathbf{r})$. The contour plot at the base is for the total $\rho_{d}(\mathbf{r})$ of the QD. (b) Difference with respect to the bulk response. $a_{0}$ is the bulk lattice constant, $a_{B}=$ $0.529 \AA$ is the Bohr radius, and the $\Delta \rho$ shown in all the figures contains only one spin, thus is half of the total response. 


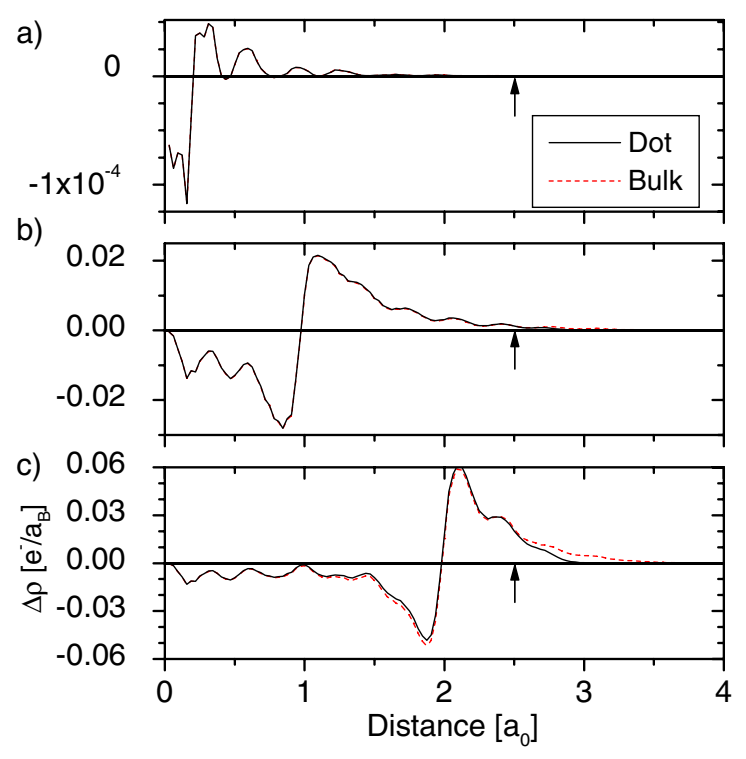

FIG. 2 (color online). Spherically averaged $\Delta \rho(\mathbf{r})$ for a 933atom GaAs dot and bulk under different perturbations. (a) $\delta$ perturbation. (b) Coulomb perturbation truncated at $a_{0}$. (c) Coulomb perturbation truncated at $2 a_{0}$. The vertical arrows indicate the dot radius $R_{d}$.

the practical equivalence of the bulk and dot responses, and see that the effect of the perturbation can be felt up to $1.5 a_{0}$ from the perturbing point, where $a_{0}$ is the bulk lattice constant $\left(a_{0}=5.65 \AA\right.$ for $\mathrm{GaAs}$, and $5.43 \AA$ for $\left.\mathrm{Si}\right)$. Note that the effective radius $R_{d}$ of the 933-atom QD computed from its total number of atoms is about $2.5 a_{0}$, as indicated by the vertical arrows in the figure. Besides the 933-atom QD, we also did the same delta perturbation for a 465-atom dot (with an effective radius of $2.1 a_{0}$ ). The result is almost the same as in Fig. 2(a), thus again indistinguishable from the bulk result.

Besides the delta function perturbation, we have also applied a weak Coulomb-like potential as the total (i.e., external + induced) perturbing potential: $\delta V_{\text {tot }}(r)=\beta / r$ ( $\beta=0.0017$ a.u.) which is truncated at $a_{0}$, so $\delta V_{\text {tot }}(r)=0$ for $r>a_{0}$. This Coulomb-like perturbing potential effectively probes source points away from the dot center. We see that in Fig. 2(b), for the 933 atom QD, the response charge is again the same as for the bulk. However, when the Coulomb potential truncation is at $2 a_{0}$, we do see a small difference between the bulk and QD charge response, especially near the boundary [Fig. 2(c)]. This is because beyond $R_{d}$ there can be no charge response from the QD. The same $2 a_{0}$ Coulomb perturbation introduces a much smaller charge response (compared to bulk) for the smaller 465atom dot, as shown in Fig. 3, because now the perturbation extends all the way to the dot boundary of $R_{d}=2.1 a_{0}$.

Besides the direct band gap GaAs QDs, we have also calculated an indirect band gap 465-atom Si QD passivated with hydrogen atoms. The situation is basically the same as for the GaAs dot: for a $\delta$ perturbation, the bulk and dot curves are the same and an $a_{0}$ truncated Coulomb pertur-

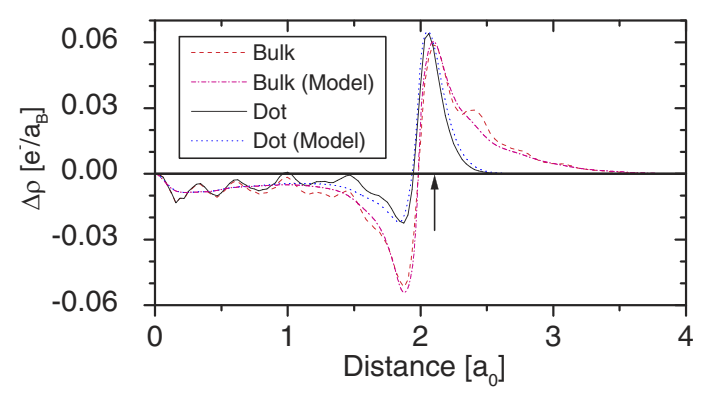

FIG. 3 (color online). As in Fig. 2, but with a smaller 465-atom GaAs dot and the Coulomb perturbation truncated at $2 a_{0}$. The Bulk and Dot are direct LDA-calculated results, and the Models are their corresponding model results according to Eq. (9). Note, in this case, the bulk and dot results are significantly different and our model predicts both of them accurately.

bation causes slight differences around the surface much like in Fig. 2(c).

Summarizing our results, we can say that when the corresponding bulk charge response of a perturbing potential does not reach the QD surface, the charge response of the dot will be the same as for the bulk. (However, near the boundary, their responses are different.) This is in clear support of the argument of Delerue et al. [5]. The screening response at the center of the QD is the same as for bulk, despite of the fact that the band gap (and thus the local density of states at the center) has changed from its bulk value. In our case, the bulk LDA band gap for GaAs is $0.66 \mathrm{eV}$, while it is 2.34 and $1.94 \mathrm{eV}$ for the $465-$ and $933-$ atom QDs, respectively. For the $\mathrm{Si}$, the bulk LDA gap is $0.67 \mathrm{eV}$, while the 465 -atom dot has a band gap of $1.71 \mathrm{eV}$.

The above observations have led us to formulate a model to calculate the microscopic susceptibility $\chi_{d}\left(\mathbf{r}_{1}, \mathbf{r}_{2}\right)$ of the dot from its bulk value $\chi_{b}\left(\mathbf{r}_{1}, \mathbf{r}_{2}\right)$. Any such a model should satisfy two requirements, namely, (i) it must be symmetric: $\chi_{d}\left(\mathbf{r}_{1}, \mathbf{r}_{2}\right)=\chi_{d}\left(\mathbf{r}_{2}, \mathbf{r}_{1}\right)$, and (ii) charge must be conserved: $\int \chi_{d}\left(\mathbf{r}_{1}, \mathbf{r}_{2}\right) d^{3} r_{2}=\int \chi_{d}\left(\mathbf{r}_{1}, \mathbf{r}_{2}\right) d^{3} r_{1}=0$. Since $\chi_{d}\left(\mathbf{r}_{1}, \mathbf{r}_{2}\right)$ must be zero when one or both of $\mathbf{r}_{1}$ and $\mathbf{r}_{2}$ is outside the QD, one simple idea is to truncate $\chi_{d}\left(\mathbf{r}_{1}, \mathbf{r}_{2}\right)$ using mask functions on $\mathbf{r}_{1}$ and $\mathbf{r}_{2}$. However, the charge conservation requirement immediately invalidates this approach. Instead, we propose to truncate the polarizability tensor

$$
\chi_{d}^{i j}\left(\mathbf{r}_{1}, \mathbf{r}_{2}, \omega\right) \equiv \frac{\delta P_{i}\left(\mathbf{r}_{1}, \omega\right)}{\delta E_{j}\left(\mathbf{r}_{2}, \omega\right)}
$$

where $P_{i}\left(\mathbf{r}_{1}, \omega\right)$ is the value of the microscopic polarization [11] due to an electric field $\mathbf{E}\left(\mathbf{r}_{2}, t\right)=\mathbf{E}\left(\mathbf{r}_{2}, \omega\right) \exp (-i \omega t)$. The tensor $\chi_{d}^{i j}\left(\mathbf{r}_{1}, \mathbf{r}_{2}, \omega\right)$ is related to a current-current response function [12], which can be calculated from the electron wave functions and current operators in a perturbation expression [12]. Our charge response function $\chi_{d}$ is just the double divergence of the tensor $\chi_{d}^{i j}$.

$$
\chi_{d}\left(\mathbf{r}_{1}, \mathbf{r}_{2}, \omega\right)=\partial_{i 1} \partial_{j 2} \chi_{d}^{i j}\left(\mathbf{r}_{1}, \mathbf{r}_{2}, \omega\right),
$$

where the symbol $\partial_{i 1}\left(\partial_{j 2}\right)$ denotes a partial derivative with 
respect to the first (second) set of position coordinates, and we have used the Einstein summation convention. Now, we provide a mask function truncating on $\chi_{d}^{i j}\left(\mathbf{r}_{1}, \mathbf{r}_{2}, \omega\right)$, leading us to

$$
\chi_{d}\left(\mathbf{r}_{1}, \mathbf{r}_{2}, \omega\right) \approx \partial_{i 1} \partial_{j 2}\left[\chi_{b}^{i j}\left(\mathbf{r}_{1}, \mathbf{r}_{2}, \omega\right) w\left(\mathbf{r}_{1}\right) w\left(\mathbf{r}_{2}\right)\right],
$$

where $w(\mathbf{r})$ is a mask function as shown in Fig. 4(a), which is effectively 1 inside the dot and zero outside with a transition region of width $w_{0}$. Equation (3) is clearly symmetric under the exchange of $\mathbf{r}_{1}$ and $\mathbf{r}_{2}$, and it satisfies the charge conservation requirement.

Since the calculation of the full microscopic bulk $\chi_{b}^{i j}\left(\mathbf{r}_{1}, \mathbf{r}_{2}, \omega\right)$ lies outside the scope of our current study, we will only test our model based on its spherically averaged properties. First, we will only test the case when $\omega=$ 0 . Second, when both $\mathbf{r}_{1}$ and $\mathbf{r}_{2}$ are inside $R_{d}-w_{0}$, according to our model, $\chi_{d}\left(\mathbf{r}_{1}, \mathbf{r}_{2}\right)$ should be the same as in the bulk. In a sense this has been verified in Fig. 2. Next, we like to test the case when one of $\mathbf{r}_{1}$ and $\mathbf{r}_{2}$ is inside $R_{d}-$ $w_{0}$, and the other one is outside. We will restrict ourselves to spherical (or $T_{d}$-symmetric) potential perturbations, and test the spherical average of the response charge. Because of the symmetry, we can assume without loss of generality that the perturbation potential at $\mathbf{r}_{2}$ is inside $R_{d}-w_{0}$ [thus $\left.w\left(\mathbf{r}_{2}\right)=1\right]$. Then we can define
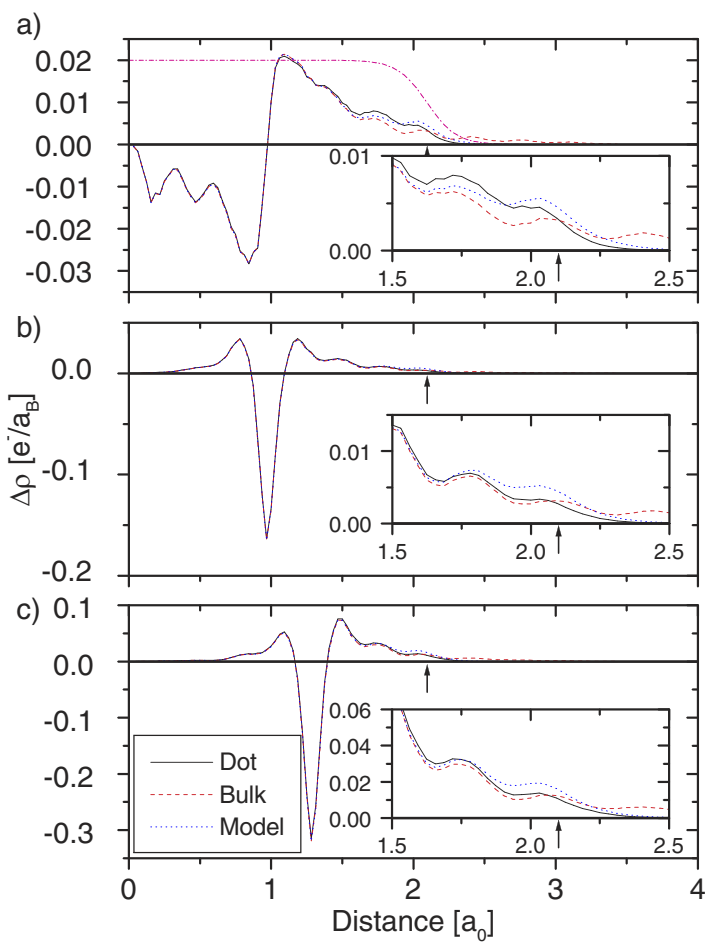

FIG. 4 (color online). As in Fig. 2, but with a 465-atom GaAs dot under a (a) Coulomb perturbation truncated at $a_{0}$; (b) $\delta\left(r_{2}-a_{0}\right)$ perturbation; and (c) $\delta\left(r_{2}-1.3 a_{0}\right)$ perturbation. The dotted line is the application of the model in Eq. (5). The insets show a close-up of the region close to the dot surface. The dashed-dotted line in (a) is the mask function $w(r)$ in Eq. (6).

$$
j_{b}\left(r_{1}\right) \equiv \int d \Omega_{1} \int \partial_{j_{2}} \chi_{b}^{\hat{r} j}\left(\mathbf{r}_{1}, \mathbf{r}_{2}\right) \Delta V\left(\mathbf{r}_{2}\right) d^{3} r_{2} .
$$

Here $\chi_{b}^{\hat{r} j}$ denotes the radial component of the first index $i$ in the tensor $\chi_{b}^{i j}$. Using Eq. (3) and our previous definition of the spherically averaged charge response $\Delta \rho_{d}\left(r_{1}\right)$, we have

$$
\Delta \rho_{d}\left(r_{1}\right)=\frac{1}{r_{1}^{2}} \frac{d}{d r_{1}}\left[r_{1}^{2} w\left(r_{1}\right) j_{b}\left(r_{1}\right)\right]
$$

Applying the same equation to bulk, we can obtain $j_{b}\left(r_{1}\right)$ for a given perturbation potential $\Delta V\left(\mathbf{r}_{2}\right)$ by integrating the LDA-calculated $\Delta \rho_{b}\left(r_{1}\right)$ assuming $w\left(r_{1}\right)=1$. Then the modeled $\Delta \rho_{d}\left(r_{1}\right)$ can be calculated from the above equation for a given $w\left(r_{1}\right)$, and can be compared with the directly calculated results. We have used a simple Fermi-Dirac-like analytical expression for the truncation function $w(r)$ :

$$
w(r)=\left[1+\exp \left(\frac{r-R_{d}}{w_{0}}\right)\right]^{-1} .
$$

The fitting parameter $w_{0}$ should be of the same order as the inverse of the Fermi vector.

Figure 4 shows the application of the model to several cases where $\mathbf{r}_{2}$ is inside $R_{d}-w_{0}$. Figure 4(a) corresponds to a Coulomb perturbation truncated at $a_{0}$ for the 465atom GaAs dot. Figure 4(b) corresponds to a $\Delta V\left(\mathbf{r}_{2}\right)=$ $\delta\left(r_{2}-a_{0}\right)$ shell perturbation, and Fig. 4(c) corresponds to a $\delta\left(r_{2}-1.3 a_{0}\right)$ shell perturbation. In our model, we have used $w_{0}=0.163 a_{0}$. Since the $R_{d}$ for this 465-atom QD is $2.1 a_{0}$, all these perturbations are roughly within $R_{d}-w_{0}$. First, at $r_{1}<R_{d}-w_{0}$ (e.g., near the origin), the QD response and the bulk response are almost the same, as predicted by our theory. In the region of $r_{1}>R_{d}-w_{0}$, there are differences between the dot and bulk responses. Here our model provides very reasonable approximations as shown in the insets. In all cases, it reproduces the transfer of the charge change from the exterior to the interior of the dot.

Because of symmetry between $\mathbf{r}_{1}$ and $\mathbf{r}_{2}$, the case of perturbing near the boundary and probing the response inside the dot has been tested in the above reverse cases. It is more complicated to model the cases where both $\mathbf{r}_{1}$ and $\mathbf{r}_{2}$ are near the boundary as in Fig. 3. For this purpose, without calculating the microscopic $\chi_{b}^{i j}\left(\mathbf{r}_{1}, \mathbf{r}_{2}\right)$, we need to make a spherical and local-field-free approximation of it as

$$
\chi_{b}^{i j}\left(\mathbf{r}_{1}, \mathbf{r}_{2}\right) \approx f_{b}\left(\left|\mathbf{r}_{1}-\mathbf{r}_{2}\right|\right) \hat{r}_{12, i} \hat{r}_{12, j},
$$

where $\hat{r}_{12, i}$ is the $i$ th component of $\hat{\mathbf{r}}_{12}=\left(\mathbf{r}_{1}-\mathbf{r}_{2}\right) /$ $\left|\mathbf{r}_{1}-\mathbf{r}_{2}\right|$. Using the above expression and Eqs. (4) and (5) for a $\delta$-like perturbation, we have

$$
\frac{d^{2}}{d r^{2}}\left[r^{2} f_{b}(r)\right]=r^{2} \Delta \rho_{b, \delta}(r)
$$

and here $\Delta \rho_{b, \delta}(r)$ is the spherically averaged bulk charge response for a $\delta$ perturbation. Using Eq. (8), the $\Delta \rho_{b, \delta}(r)$ shown in Fig. 2(a), and zero value boundary conditions for 
$r \rightarrow \infty$, we can solve for $f_{b}(r)$. Then, Eq. (7) can be used in Eq. (3), and the charge response to a spherical perturbing potential $v(r)$ in a $\mathrm{QD}$ can be calculated as

$$
\begin{aligned}
\Delta \rho\left(r_{2}\right)= & \frac{2 \pi}{r_{2}^{2}} \frac{d}{d r_{2}}\left\{r _ { 2 } ^ { 2 } w ( r _ { 2 } ) \int \int \left[\left(\frac{1}{r_{12}^{2}} \frac{d}{d r_{12}} r_{12}^{2} f_{b}\left(r_{12}\right)\right) w\left(r_{1}\right)\right.\right. \\
& \left.\left.-f_{b}\left(r_{12}\right) \frac{z_{12}}{r_{12}}\left(\frac{d}{d r_{1}} w\left(r_{1}\right)\right)\right] \frac{z_{21}}{r_{12}} v\left(r_{1}\right) r_{1}^{2} \sin \theta d \theta d r_{1}\right\},
\end{aligned}
$$

where $r_{12}=\sqrt{\left(r_{2}-r_{1} \cos \theta\right)^{2}+r_{1}^{2} \sin ^{2} \theta}, z_{12}=r_{1}-r_{2} \cos \theta$, $z_{21}=r_{2}-r_{1} \cos \theta$, and $\theta$ is the angle between $\mathbf{r}_{1}$ and $\mathbf{r}_{2}$; the integral for $r_{1}$ goes from 0 to $\infty$, and the integral for $\theta$ is from 0 to $\pi$. Using the above equation, the charge response for any given spherical perturbation $v(r)$ can be numerically calculated. The bulk response for the $2 a_{0}$-truncated Coulomb perturbation is calculated using Eq. (9) with $w(r)=1$ and plotted in Fig. 3 (bulk model). We see that this model result is extremely close to the direct LDAcalculated charge response. This indicates that our spherical and local-field-free approximation is a reasonable approximation. We then calculated the response in the 465atom QD using the truncation function $w(r)$ of Eq. (6). The result is shown in Fig. 3. Again, we see excellent agreement between the model result and the direct LDA result, although the QD results are significantly different from the bulk results in this case. This demonstrates the accuracy of our QD microscopic dielectric response function of Eq. (3).

Another way to check our model is to calculate the overall averaged QD dielectric constant. This constant can be defined in different ways [1]. The dielectric constant calculated from its total polarization [13] under a constant electric field is calculated directly using LDA as 5.2 for the 465-atom GaAs dot, compared with its 12.8 value for the bulk. The overall dielectric constant for the spherical exciton screening as defined in Ref. [1] is 3.6 and 4.6 for the 465-atom and 933-atom GaAs QDs, respectively, calculated directly using LDA. The spherical charge response of this problem can also be calculated by Eq. (9); the resulting dielectric constants are 3.9 and 4.3 for these two QDs, which are close to the directly calculated results.

So far our tests are based on spherical perturbations. The model in Eq. (3) is general, and it can be used for any perturbation. In Fig. 5, we show the LDA charge response of the 465-atom GaAs QD to an off-center $\delta$ perturbation. At the perturbing point, the QD response is almost the same as the bulk response (the difference is only $\sim 1 \%$ ). This is consistent with the fact that the perturbing point is slightly inside the $R_{d}-w_{0}$ region. Away from the perturbing point, the biggest difference comes from the region near the QD boundary (about 100\% difference from the bulk value). All these agree qualitatively with our model,

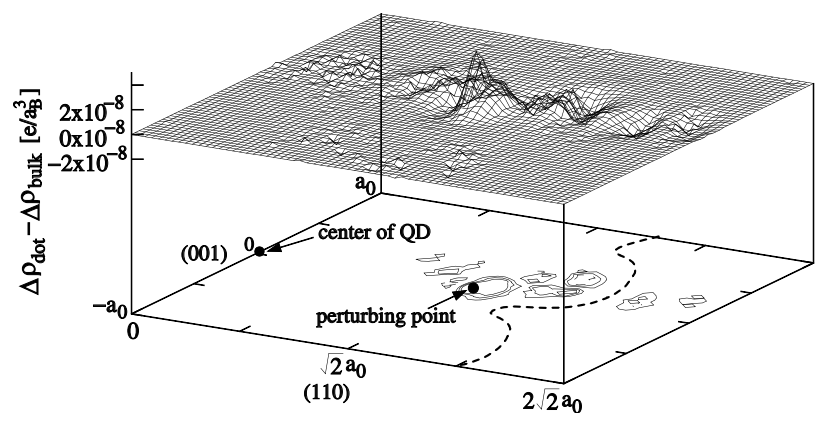

FIG. 5. Off center $\delta$-like perturbation (same amplitude as in Fig. 1) for the 465-atom GaAs quantum dot. Note the scale, the $\Delta \rho_{\text {dot }}-\Delta \rho_{\text {bulk }}$ is bigger than that in Fig. 1(b). The thick dashed line in the lower panel contour plot indicates the boundary of the QD as the total QD charge density drops to $4 \%$ of its bulk average. The $R_{d}$ of this dot is $2.1 a_{0}$. Only a small portion of the (110) cross section is shown here.

Eq. (3). Unfortunately, without calculating the full microscopic $\chi_{b}^{i j}\left(\mathbf{r}_{1}, \mathbf{r}_{2}\right)$, a more quantitative test for this nonspherical case is not possible at this time.

Note that we have discussed $\chi\left(\mathbf{r}_{1}, \mathbf{r}_{2}\right)$ in this Letter, which can be used to solve the Poisson equation. Especially, using the approximation of Eq. (7), fast numerical techniques (e.g., the fast fourier transformation) can be used. Our model for $\chi\left(\mathbf{r}_{1}, \mathbf{r}_{2}\right)$ [hence $\epsilon\left(\mathbf{r}_{1}, \mathbf{r}_{2}\right)$ ] could also provide the first step for the modeling of $\epsilon^{-1}\left(\mathbf{r}_{1}, \mathbf{r}_{2}\right)$ [14] of a QD, which is used in formalisms like the GW method.

We would like to thank Dr. A. Franceschetti for stimulating discussions about this problem. This work was supported by the Department of Energy under Contract No. DE-AC03-76SF00098; it uses the resources of the National Energy Research Scientific Computing Center.

[1] L.-W. Wang and A. Zunger, Phys. Rev. Lett. 73, 1039 (1994).

[2] R. Tsu and D. Babic, Appl. Phys. Lett. 64, 1806 (1994).

[3] M. Lannoo et al., Phys. Rev. Lett. 74, 3415 (1995).

[4] T. D. Krauss and L.E. Brus, Phys. Rev. Lett. 83, 4840 (1999); 84, 1638(E) (2000).

[5] C. Delerue et al., Phys. Rev. B 68, 115411 (2003).

[6] M. von Laue, Ann. Phys. (Leipzig) 44, 1197 (1914).

[7] C. Kittel, Quantum Theory of Solids (Wiley, New York, 1963), 1st ed.

[8] G. Allan et al., Phys. Rev. B 52, 11982 (1995).

[9] V. A. Fonoberov et al., Phys. Rev. B 66, 85310 (2002).

[10] L.-W. Wang and J. Li, Phys. Rev. B 69, 153302 (2004).

[11] L. L. Hirst, Rev. Mod. Phys. 69, 607 (1997).

[12] S. L. Adler, Phys. Rev. 126, 413 (1962).

[13] I. Vasiliev et al., Phys. Rev. Lett. 78, 4805 (1997).

[14] S. Öğüt et al., Phys. Rev. Lett. 90, 127401 (2003). 